\title{
Anisotropic, gradient and metal-like mechanical behaviors of teeth and their implications on tooth functions
}

\author{
BAI Ke ${ }^{1}$, ZHANG TaiHua ${ }^{2}$, YANG ZhiYu ${ }^{1}$, SONG Fan ${ }^{2}$, YANG XiaoDa ${ }^{3} \&$ WANG $\mathrm{Kui}^{3 \dagger}$ \\ ${ }^{1}$ School of Pharmaceutical Science, Peking University Health Science Centre, Beijing 100083, China; \\ ${ }^{2}$ State Key Laboratory of Nonlinear Mechanics (LNM), Institute of Mechanics, Chinese Academy of Sciences, Beijing 100080, \\ China; \\ ${ }^{3}$ State Key Laboratory of Natural and Biomimetic Drugs, School of Pharmaceutical Sciences, Peking University Health Science \\ Center, Beijing 100083, China
}

The anisotropy and gradient of the elastic modulus and the hardness of teeth were investigated by means of instrumented indentation method. Such properties are attributed to the unique microstructures of teeth based on scanning electron microscopic analysis. By comparing the relationship between the ratio of hardness to the reduced elastic modulus and the ratio of elastic unloading work to the total work of teeth in course of indentation to those of other materials, we found that the material behaviors of teeth display metal-like characteristics rather than ceramics as considered traditionally. These material behaviors and relevant functions are discussed briefly.

tooth, mechanical properties, instrumented indentation, gradient, anisotropic, metal-like

The most important and impressive function of teeth is life-long mastication ${ }^{[1,2]}$. This implies that the structural and mechanical properties of the tooth materials can sustain repetitive occlusal loading for tens years. Teeth are highly mineralized tissues with micro- and nanostructural features and the arrangement of these features can significantly affect the mechanical properties ${ }^{[1]}$. Therefore, to understand the superior microstructures and mechanical properties of teeth are important not only in clinical practice but also in physical and materials sciences.

Recently, a lot of mechanical tests on teeth were performed with instrumented indentation or nanoindentation method. Cuy et al. made a nanoindentation mapping of the mechanical properties of human molar tooth enamel and presented that the material behaviors of enamel were not homogeneous ${ }^{[2]}$. Marshall et al. found that mechanical properties through the normal dentin remained relatively constant and the transition between enamel and dentin was smooth ${ }^{[3,4]}$. At even smaller scales, the hardness and Young's modulus of single apatite crystal rods in enamel were measured with nanoindentation $^{[5]}$. More recently, Nizam and Lim indicated that although nanoindentation on teeth is a relatively new area of research, it provides an excellent way to probe and relate the structures and mechanical properties of teeth at the submicron and nanometer scales that were previously not possible but can now greatly benefit dental research. For example, tooth enamel is made up of the hydroxylapatite crystal rods and their orientations will affect the nano-mechanical properties obtained. Thus, anisotropy of teeth remains an interesting area of research $^{[1]}$.

In the present study, by means of nanoindentation method, we examine the elastic modulus, the hardness

\footnotetext{
Received February 12, 2007; accepted March 15, 2007 doi: 10.1007/s11434-007-0368-9

${ }^{\dagger}$ Corresponding author (email: wangkui@bjmu.edu.cn)

Partly supported by the National Natural Science Foundation of China (Grant Nos. 10572142 and 10672164)
} 
of teeth, the ratio of hardness to reduced elastic modulus, and the ratio of elastic unloading work to the total work of enamel and dentin in course of nanoindentation testing. It is found that the mechanical properties of teeth are not only anisotropic but also gradient. Also, the crack characteristics on the dentin-enamel junction (DEJ) are discussed and analyzed. More significantly, the relationship between the ratio of reduced elastic modulus to hardness and the ratio of elastic unloading work to the total work shows that teeth have metal-like behaviors rather than ceramics as considered traditionally. Finally, we demonstrate that these material and mechanical properties play key roles in teeth functions, such as the matching between the enamel and dentin, as well as their life-long mastication.

\section{Materials and methods}

The tested samples of teeth were taken from molars and canines. All the teeth were removed in less than 12 month. Once the teeth were removed from the mouth, they were enwrapped in the gauze immersed with $20 \%$ glycerin and were stored at $4^{\circ} \mathrm{C}$. This storage condition has been widely used and provides the essential humidity and temperature which prevents the teeth from dryness, crack, and decomposition ${ }^{[5]}$. We chose some cross sectional area to examine the mechanical and microstructural properties of teeth, as shown in Figure 1. The sectioned samples of teeth were mounted with denture base resin (Type II ) and epoxy resin, and all the tested surfaces of the samples were carefully polished.

In order to measure the mechanical properties of teeth, indentation experiments were carried out using the MTS Nano Indenter XP with a Berkovich diamond tip to test the surface of each sample. Load and displacement resolution of MTS Nano Indenter XP were controlled to be within $50 \mathrm{nN}$ and $0.01 \mathrm{~nm}$, respectively. In addition, the relevant microstructures of the tested samples were investigated with a scanning electron microscope (SEM, Sirion 400NC) and all tested data of SEM images were automatically analyzed and recorded by employing Image Analysis System (IAS, Image-Pro Plus 4.5, Media Cybernetics).

Based on the Oliver-Pharr method ${ }^{[6,7]}$, the reduced elastic modulus, $E_{r}$, can be evaluated from the nanoindentation measurements by the following equation:

$$
E_{r}=\frac{\sqrt{\pi}}{2 \beta} \frac{S_{u}}{\sqrt{A}},
$$

where $A$ is the contact area at maximum load which can be calculated by the indenter shape function, $S_{u}$ is the contact stiffness, and $\beta=1.034$ is constant in OliverPharr method. The hardness $H$ can be obtained from the following relation:

$$
H=\frac{P_{m}}{A},
$$

here $P_{m}$ is the peak load.

The effects of a non-rigid indenter on the load displacement behavior can be taken into account by the reduced modulus $E_{r}$ as follows:

$$
\frac{1}{E_{r}}=\frac{1-v^{2}}{E}+\frac{1-v_{i}^{2}}{E_{i}},
$$

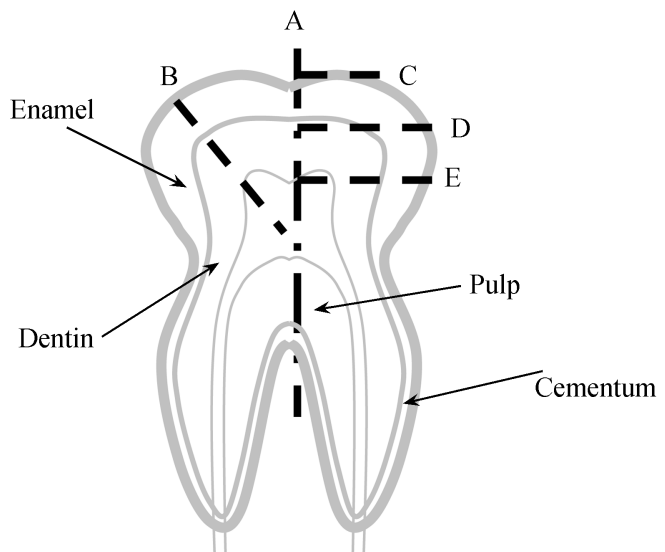

(a)

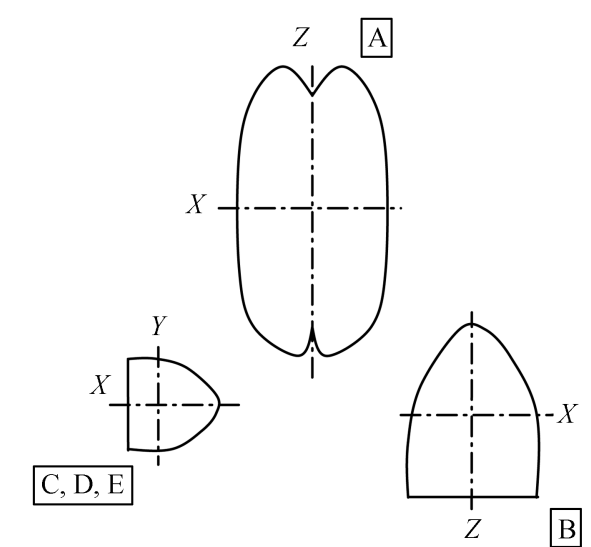

(b)

Figure 1 The anatomic sketch of the third molar shows the positions of the cross sectional area tested and the directions of nanoindentation. (a) The positions of sections A, B, C, D and E in the tooth; (b) the tested direction of each section. 
where $E$ is elastic modulus, $v$ is Poisson's ratio of the sample tested, and $E_{i}$ and $v_{i}$ are the corresponding values of the indenter. For the diamond indenter used in our experiments, $E_{i}=1141 \mathrm{GPa}$ and $v_{i}=0.07$. Also, in all calculation, Poisson's ratio of the specimen was assumed to be 0.25 . Furthermore, in the course of nanoindentation testing, the maximum indentation depth was controlled to be one micrometer so that the trace made by Berkovich tip was an equilateral triangle with a side length of approximately $7 \mu \mathrm{m}$. Note that the distance of adjacent indents was set to be twenty times greater than that of the indent to avoid the overlapping of influence regions of indents.

\section{Results and discussion}

Three types of loading and unloading curves of the nanoindentation on teeth under the same testing conditions are shown in Figure 2. The curve of dentin is normal and smooth with maximum load about $20.5 \mathrm{mN}$. However, the situations of enamel are different when the positions of the elected sections are different. One curve that describes the course of the testing on section A or B is no longer as smooth as that of dentin and shows clear pop-in, which indicates that micro-cracking occurs around the indent tips. The other that describes the course of the testing on section $\mathrm{C}$ or D or $\mathrm{E}$ still remains smooth similar to that of the dentin, which testifies that micro-cracking does not occur. These prove that the mechanical properties of enamel are anisotropic.

Further, we examined the variations of the average modulus and the average hardness on the different positions and directions of the sections elected previously, as shown in Table 1. The tested results demonstrate that the mechanical properties of enamel are anisotropic, while the properties of dentin are approximately isotropic.

In addition, a typical plot of the results is shown in Figure 3. It shows the variation of modulus and hardness

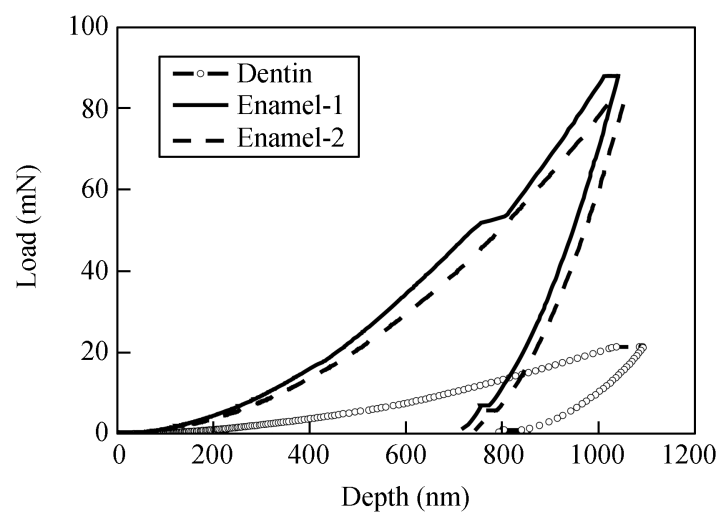

Figure 2 Curves of load $P$ against indentation depth $h$ showing the loading and unloading loops.

along $X$ and $Y$ directions on section $\mathrm{C}$ in enamel. Both modulus and hardness present some differences between $X$ and $Y$ directions. In particular, the diagram shows a valley-shaped variation. As shown in Figure 1(a), section $\mathrm{C}$ is perpendicular to the vertical $Z$, thus it is roughly parallel to the occlusal surface of tooth. However, since the rough occlusal surface of that part is convex outward, the measurements made on section $\mathrm{C}$ represent some variations from its outmost surface to its inner part of enamel. This displays a well-organized gradient property from the surface to the inside of enamel.

The hydroxylapatite crystals, which are the main components of the enamel, are in the form of rods with diameter of $4-6 \mu \mathrm{m}^{[2]}$ and stand upright on the surface of dentine and run through the whole thickness of enamel ${ }^{[8]}$. However, by employing scanning electronic microscopy (SEM), we observed that the crystaline rods near the occlusal surface (Figure 4(a)) are short and thick while the rods near the DEJ (Figure 4(b)) are long and thin, which is in accord with the gradient variations of modulus and hardness in the enamel stated above. This is an indication that the gradient of the mechanical properties of enamel should derive from its microstructure.

Table 1 The data of elastic modulus, hardness and $W_{e} / W_{t}$ of sections A, B, C, D and E

\begin{tabular}{|c|c|c|c|c|c|c|c|c|c|c|}
\hline \multirow{2}{*}{\multicolumn{2}{|c|}{ Direction of section }} & \multicolumn{5}{|c|}{ Enamel } & \multicolumn{4}{|c|}{ Dentin } \\
\hline & & \multirow{2}{*}{$\frac{\text { vertical section }}{\mathrm{A}}$} & \multicolumn{3}{|c|}{ horizontal section } & \multirow{2}{*}{$\begin{array}{c}\begin{array}{c}\text { inclined } \\
\text { section }\end{array} \\
\mathrm{B}\end{array}$} & \multirow{2}{*}{$\begin{array}{c}\begin{array}{c}\text { vertical sec- } \\
\text { tion }\end{array} \\
\text { A }\end{array}$} & \multirow{2}{*}{$\begin{array}{c}\begin{array}{c}\text { inclined } \\
\text { section }\end{array} \\
\mathrm{B}\end{array}$} & \multicolumn{2}{|c|}{ horizontal section } \\
\hline \multicolumn{2}{|c|}{ Section } & & $\mathrm{D}$ & $\mathrm{E}$ & $\mathrm{C}$ & & & & $\mathrm{D}$ & $\mathrm{E}$ \\
\hline \multirow{2}{*}{$\begin{array}{l}\text { Modulus } \\
(\mathrm{GPa})\end{array}$} & $Z / Y$ & 81.02 & 83.39 & 91.93 & 97.59 & 82.57 & 24.11 & 24.36 & 23.55 & 23.34 \\
\hline & $X$ & 80.56 & 83.06 & 95.83 & 98.88 & 66.67 & 22.77 & 22.26 & 24.18 & 24.49 \\
\hline \multirow{2}{*}{$\begin{array}{l}\text { Hardness } \\
\text { (GPa) }\end{array}$} & $Z / Y$ & 4.11 & 4.02 & 4.27 & 4.98 & 3.93 & 0.85 & 0.87 & 0.85 & 0.88 \\
\hline & $X$ & 4.13 & 4.04 & 4.53 & 4.87 & 3.83 & 0.81 & 0.81 & 0.85 & 0.89 \\
\hline \multirow{2}{*}{$\begin{array}{l}W_{e} / W_{t} \\
(\%)\end{array}$} & $Z / Y$ & 31.37 & 30.64 & 33.31 & 31.37 & 30.84 & 25.53 & 26.15 & 26.01 & 26.53 \\
\hline & $X$ & 32.03 & 30.45 & 34.97 & 31.54 & 33.65 & 25.20 & 25.032 & 25.50 & 26.58 \\
\hline
\end{tabular}



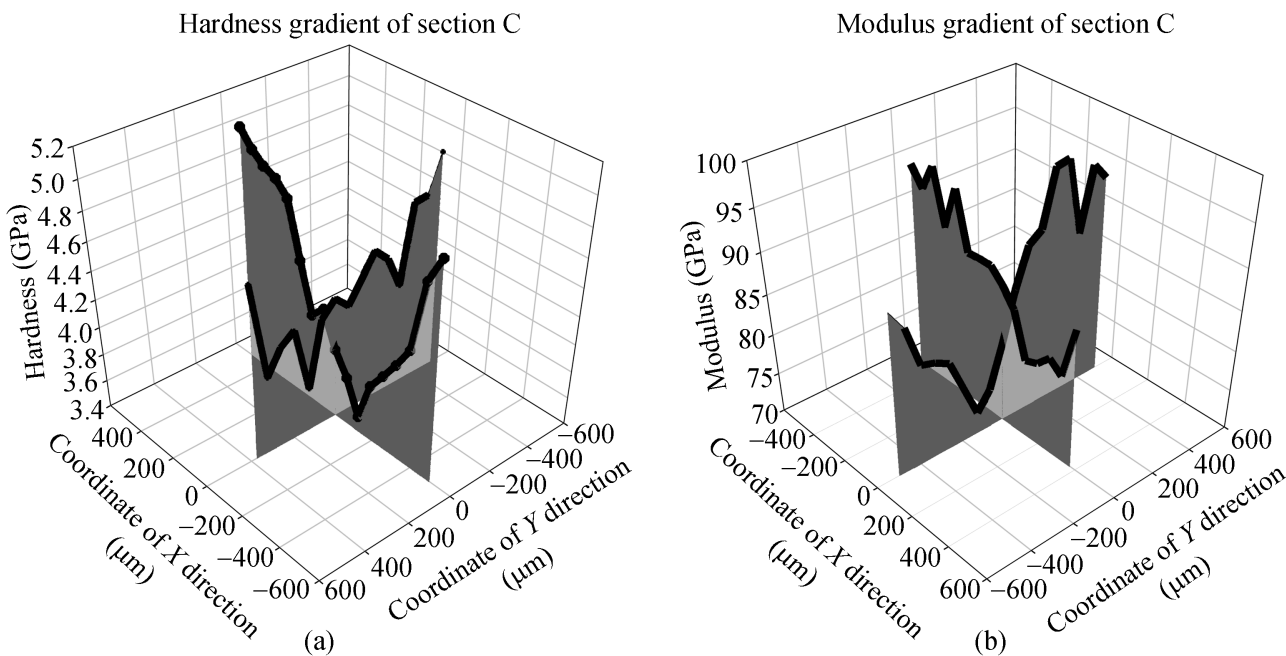

Figure 3 Plots showing variations of the modulus and hardness on section $\mathrm{C}$ in enamel. The valley-shaped distributions show the gradient decreasing of modulus and hardness from the outmost surface and the anisotropy in $X$ and $Y$ directions in enamel. In this figure, origin is the cross of $X$ and $Y$ directions. The positive numbers of $X$ and $Y$ directions indicate the left side from the origin to the edge of section $\mathrm{C}$ and the negatives indicate the right side from the origin to the edge of section $\mathrm{C}$.
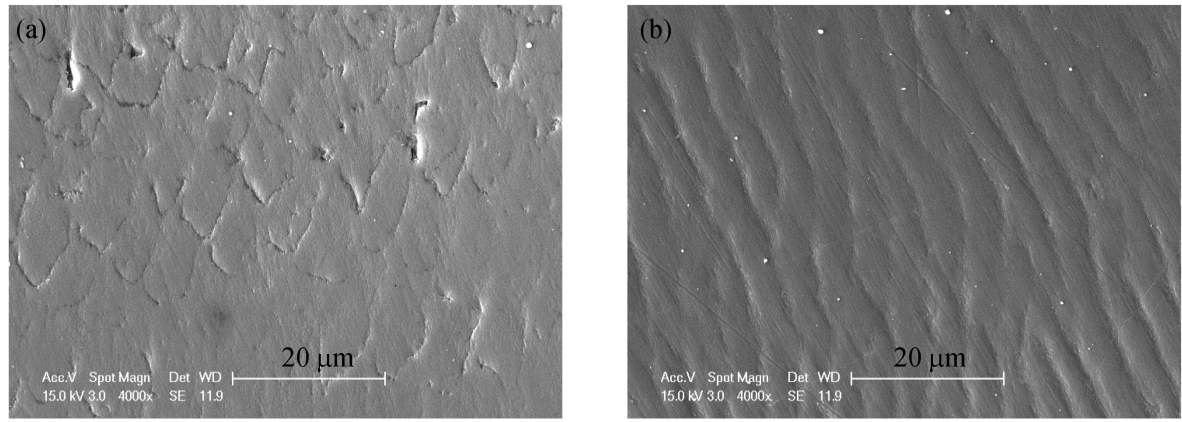

Figure 4 SEM images showing microstructural variations of enamel.

SEM images show that the microscopic flaws initiate from DEJ on the enamel side but no such micro-flaws on dentin side, as shown in Figure 5. These micro-cracks appear to spread into enamel to some extent. We measured the distribution of the length of these micro-cracks and gave the average length to be about $175 \mu \mathrm{m}$. Further, we coarsely measured the chewing forces with foil pressure sensors (Digital Multimeter with Dynasen's Carbon Gauge) and obtained the average chewing pressure to be about 6.6 MPa for male and 5.2 MPa for female. Based on these measurements, we evaluated the tolerance stress $\sigma_{F}$ in terms of elastic fracture mechanics by $\sigma_{F} \approx K_{C} / \sqrt{\pi a}$. By taking the toughness of enamel to be 0.52 to $0.76 \mathrm{MPa} \cdot \mathrm{m}^{1 / 2[3,4]}$ and microcrack length $a$ $\sim 175 \mu \mathrm{m}$, the tolerance stress $\sigma_{F}$ would be 25 to $33 \mathrm{MPa}$, which is much greater than the average chewing force measured. This implies that the observed micro-flaws can be safely tolerated in enamel, unless comparatively longer cracks are created from the outmost surface under external loading and propagate through enamel. Therefore, the existence of these micro-cracks not only demonstrates the additional anisotropic feature of enamel, but can also provide a proper tolerance to release the stress. In fact, the difference between the elastic modulus of enamel and dentin can induce high internal stress, since the teeth work under repetitive occlusal loading of $10^{3}$ cycles/day and $20 \mathrm{MPa}^{[9,10]}$. Besides the cyclic pressure loading, the enamel also takes the function of cutting and grinding food with minimal wear through the life time. However, the physiological and mechanical functions of these anisotropic features of enamel have not been understood very well yet. 

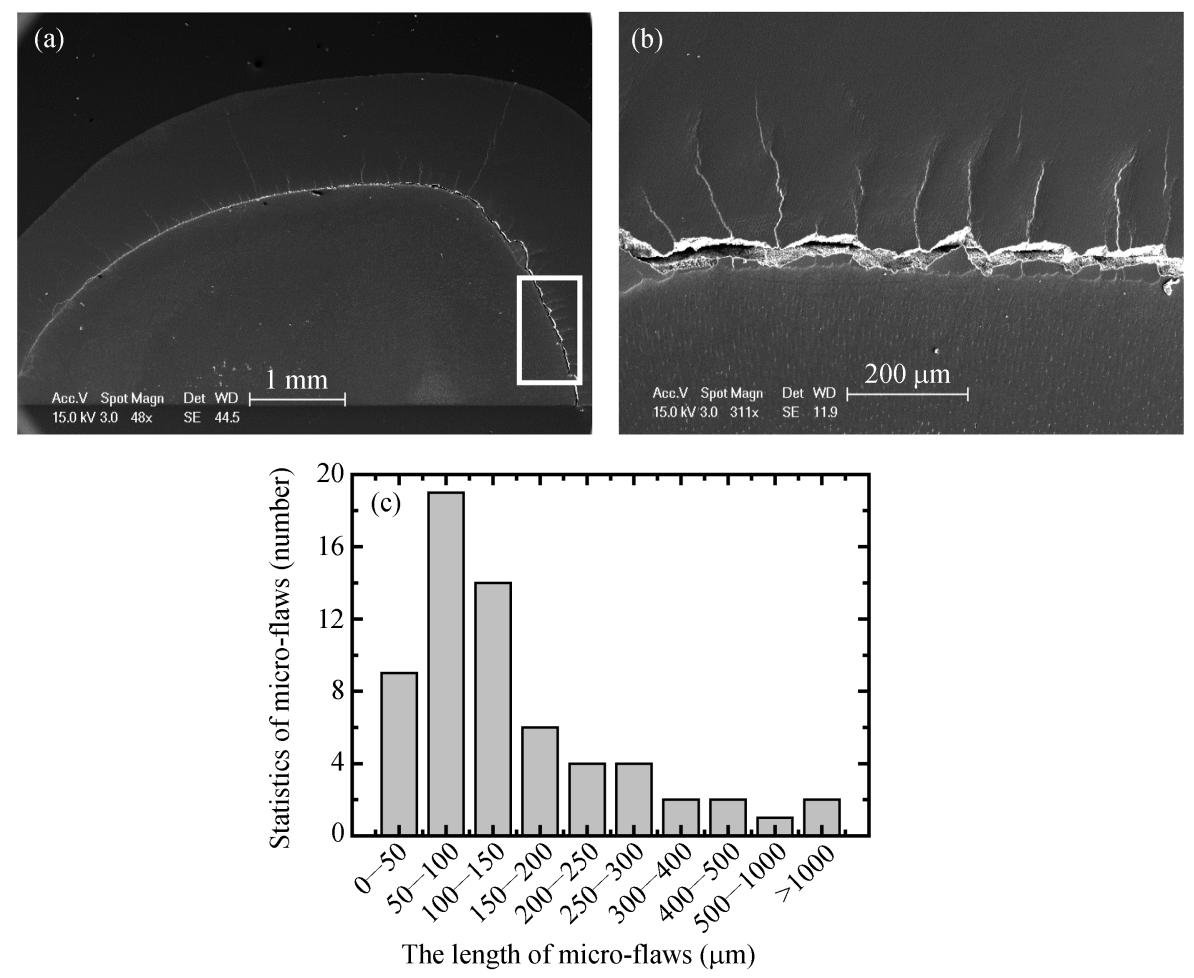

Figure 5 (a) SEM image showing DEJ in tooth; (b) a part in (a) showing micro-flaws that initiate from DEJ and extend into enamel; (c) a histogram showing statistics of the length of micro-flaws.

In particular, we studied the relationship between the ratio of hardness to reduced elastic modulus $H / E_{r}$ and the ratio of unloading work to total work $\zeta=W_{e} / W_{t}$. The averaged values of $\zeta$ were measured to be about 0.326 and 0.262 in enamel and dentin, respectively, as shown in Table 1. This indicates that the indents in enamel involve more elastic deformation than those in dentin. Interestingly, the data of many materials collapse nearly on a straight line in the diagram (see Figure 6), as suggested in ref. [11]. The datum of dentin $(\zeta=0.264)$ locates in the region where steel (for $\mathrm{GCr} 15$ steel $\zeta=0.287$ in Figure 6) and Ti alloy (for Ti alloy $\zeta=0.230$ in Figure 6) do. Unexpectedly, the datum of enamel $(\zeta=0.326)$ locates at where bulk metallic glasses (BMG, for La-based $\mathrm{BMG} \zeta=0.308$ and CuHf-based BMG $\zeta=0.317$ in Figure 6) do, rather than brittle ceramics (see $\mathrm{Al}_{2} \mathrm{O}_{3}$, glass and fused silica in Figure 6). Recalling the great difference between the fracture toughness, like $K_{I C}=$ $70-100 \mathrm{MPa} \mathrm{m}^{1 / 2}$ for steel whilst 0.52 to $0.76 \mathrm{MPa} \mathrm{m}^{1 / 2}$ for enamel ${ }^{[3,4]}$, the ratios of $H / E_{r}$ and $\zeta$ similar to steel and metallic glasses in dentin and enamel are very impressive. In fact, the ratio of $H / E_{r}$ is related to the deformation properties of a rough surface to govern wear resistance in tribology ${ }^{[12]}$ and the ratio of reversible work to total work $\zeta$ is the indication of energy dissipation. So, the relatively same position of enamel and metallic glasses in the diagram of $H / E_{r}$ vs $\zeta$ (Figure 6) indicates that the enamel presents similar balanced functions in both the resistance against wearing and the energy dissipation as metals, rather than ceramics as people usually supposed.

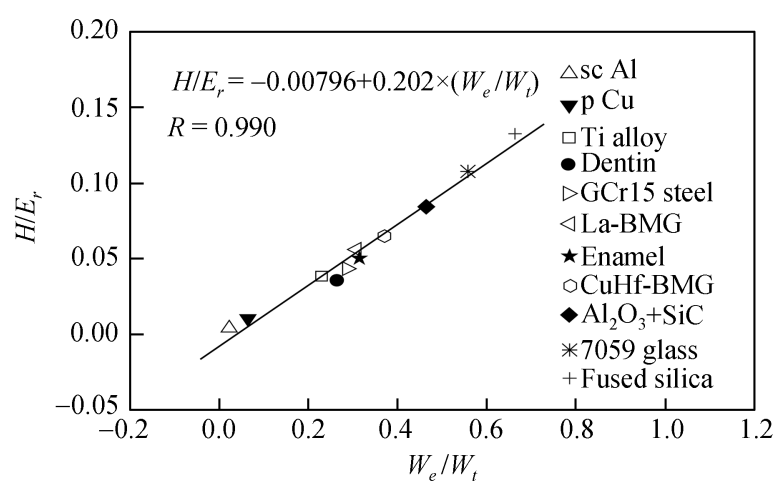

Figure 6 The plot showing the relationship between the ratio of hardness to reduced elastic modulus $H / E_{r}$ and the ratio of unloading work to total work $W_{e} / W_{t}$. The value of enamel is located in the range of metallic glasses (La-based BMG and CuHf-based BMG) and the value of dentin is located in the range of Ti alloy and GCr15 steel. 


\section{Summary}

Nanoindentation provides an excellent way to understand the relationship between the microstructures and mechanical properties of teeth. By means of instrumented indentation method, we report that the elastic modulus and hardness of enamel are not only anisotropic but also gradient. In combination with SEM observations, these special anisotropic features are attributed to the microstructures of enamel. In particular, $H / E_{r}$ and $W_{e} / W_{t}$ of dentin and enamel are similar to steel and metallic glasses, respectively, rather than ce-

1 Hairul Nizam B R, Lim C T. Nanoindentation of teeth_A review. J Exp Mech, 2006, 21(1): 35-50

2 Cuy J L, Mann A B, Livi K, et al. Nanoindentation mapping of the mechanical properties of human molar tooth enamel. Arch Oral Bio, 2002, 47: 281-291

3 Marshall G W, Balooch Jr M, Gallagher R R, et al. Mechanical properties of the dentinoenamel junction: AFM studies of nanohardness, elastic modulus, and fracture. J Biomed Mater Res, 2001, 54: 87-95

4 Marshall S J, Balooch M, Habelitz S, et al. The dentin-enamel junction-A natural, multilevel interface. J Euro Cera Soc, 2003, 23: 2897-2904

5 Habelitz S, Marshall S J, Marshall GW Jr, et al. Mechanical properties of human dental enamel on the nanometer scale. Arch Oral Bio, 2001, 46(2): $173-183$

6 Oliver W C, Pharr G M. An improved technique for determining hardness and elastic modulus using load and displacement sensing ramics. These metal-like behaviors imply that teeth have balanced functions in both the resistance against wearing and the energy dissipation, similar to metals. Enamel is the outmost layer of tooth suffering from complex loadings and environment. So, these behaviors of teeth should play key roles in their functions. The material properties need even further study in the future.

The authors are grateful to Prof. Shen L T, Prof. Li N, Dr. Xie J J, Mr. Li D $Y, M s$. Deng $Y L, M r$. Yang $R$ and Mr. Fu Y for their kind helps in the experimental measurements. Also the authors are indebted to Wuhan Hospital of Stomatology for providing the human teeth samples.

indentation experiments. J Mater Res, 1992, 7(1): 1564—1583

7 Zhang T H. Techniques and Applications of Micro/nano-mechanical Measurements (in Chinese). Beijing: China Machine Press, 2004

8 Fawcett D W. A Textbook of Histology. 11st ed. Philadelphia: W B Saunders, 1986

9 Cui F Z, Feng Q L. Bio-materials (in Chinese). Beijing: Science Press, 1996

10 Ge J, Cui F Z, Wang X M. Property variations in the prism and the organic sheath within enamel by nanoindentation. Biomat, 2005, 26(16): 3333-3339

11 Yang-Tse Cheng, Che-Min Cheng. Relationships between hardness, elastic modulus, and the work of indentation. Appl Phys Lett, 1998, 73(53): $614-616$

12 Ni W Y, Cheng Y T, Lukitsch M J, et al. Effects of the ratio of hardness to Young's modulus on the friction and wear behavior of bilayer coatings. Appl Phys Lett, 2004, 85(18): 4028—4030 